\title{
Negative Chemotaxis of Zoospores of the Fungus Phytophthora palmivora
}

\author{
By J. NEIL CAMERON† AND MICHAEL J. CARLILE* \\ Department of Biochemistry, Imperial College of Science and Technology, \\ London $S W 72 A Z$
}

(Received 24 January 1980; revised 3 April 1980)

\begin{abstract}
Phytophthora palmivora zoospores are repelled by many low molecular weight cations. This negative chemotactic response occurs at a different threshold concentration for each cation, that for the most effective ion, $\mathrm{H}^{+}$, being $150 \mu \mathrm{M}$. The effectiveness of different cations parallels their ionic conductivities, the most active cations having the highest conductivities. It is suggested that the close approach of cations to the cell surface reduces the negative charge at the surface and hence changes the transmembrane potential, altering flagellum activity in such a way as to cause turning and negative chemotaxis.
\end{abstract}

\section{INTRODUCTION}

Zoospores of Phytophthora palmivora (Butler) Butler, an important plant pathogenic fungus, tend to swim upwards, i.e. to show negative geotaxis (Cameron \& Carlile, 1977). They are also attracted by root exudates and pod extracts of a major host, cocoa, and by various pure substances produced or likely to be produced by the host (Cameron \& Carlile, 1978). Phytophthora cinnamomi zoospores also show positive chemotaxis but in addition are repelled by various cations (Allen \& Harvey, 1974). We here show that this negative chemotaxis occurs in $P$. palmivora, confirm Allen \& Harvey's conclusion that the repulsion is a threshold response taking place at a critical concentration characteristic of each cation, and suggest a possible mechanism for the response.

\section{METHODS}

Production of zoospores. The strain of Phytophthora palmivora, routine culture, media and production of zoospores were as described previously (Cameron \& Carlile, 1978). The pH 5.8 buffered medium in which the

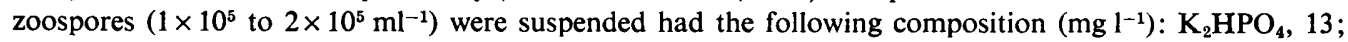
$\mathrm{KH}_{2} \mathrm{PO}_{4}, 105 ; \mathrm{MgCl}_{2} \cdot 6 \mathrm{H}_{2} \mathrm{O}, 10 ; \mathrm{CaCl}_{2} \cdot 2 \mathrm{H}_{2} \mathrm{O}, 7$; distilled water. The organism was used under licence from the Ministry of Agriculture, Fisheries and Food.

Chemotaxis assay chambers. A block of clear acrylic plastic (Perspex; $48 \mathrm{~mm} \times 48 \mathrm{~mm} \times 5 \mathrm{~mm}$ deep) was machined to produce six assay chambers on its upper surface. The chambers were $20 \mathrm{~mm} \times 6 \mathrm{~mm} \times 1 \mathrm{~mm}$ deep and from the end of each chamber a groove extended to the edge of the block to facilitate the insertion of a capillary tube into the chamber. After filling with test or control solutions the chambers were covered with a large coverslip.

Assessment of negative chemotaxis. The method used was based on that of Allen \& Harvey (1974). A zoospore suspension was drawn into a capillary tube ( $5 \mu \mathrm{l}$; Microcaps, Drummond Scientific Co., U.S.A.) and one end of the tube was sealed with petroleum jelly (Vaseline), leaving an air gap. The open end of the tube was then inserted at room temperature $\left(22 \pm 1^{\circ} \mathrm{C}\right)$ into the chemotaxis chamber which contained either the buffer used for suspending zoospores (control) or a salt to be tested dissolved in the buffer. The substance under test diffused into the capillary tube, and if it was repellent, the zoospores retreated along the tube, leaving a region nearly free from zoospores, a dense band of zoospores and a region with zoospores at the

$\dagger$ Present address: BBC Open University Production Centre, Alexandra Palace, London N22. 
initial density. The distance $x$ from the mouth of the tube to the boundary between the zoospore-free region and the dense band of zoospores was recorded with a microscope vernier to the nearest $0.1 \mathrm{~mm}$ at a series of times $t$. Five or more replicates were used for each chemical tested and the mean value and standard deviation of $x$ was calculated for each time of observation (Snedecor \& Cochran, 1974).

Mathematical description. Our treatment is an extension of that of Allen \& Harvey (1974). The diffusion coefficient $D$ of each chemical was assumed to be constant, an approximation necessary because of limited information on the variation of $D$ with concentration $C$ and to simplify calculation. The approximation is unlikely to have introduced serious error, but may be responsible for a minor discrepancy noted below. Values of the diffusion coefficients for the salts tested were obtained from Weast (1976), and that of $\mathrm{HCl}$ from Allen \& Harvey (1974) to permit comparison with their work. Diffusion in one dimension over the distance $x$ for various times $t$ with constant $D$ is described for various concentrations $C$ by Fick's second law of diffusion,

$$
\partial C / \partial t=D \partial^{2} C / \partial x^{2}
$$

Analytical solutions are obtainable (Crank, 1975) for the boundary conditions pertaining to the negative chemotaxis assay, i.e.

$$
C=C_{0}, x<0 ; \quad C=0, x>0 ; \quad t=0
$$

At time $t$ the concentration $C$ at $x \mathrm{~mm}$ from the open end of the tube is given by

or

$$
\begin{gathered}
C(x, t)=C_{0} \operatorname{erfc} 0.5 x(D t)^{-0.5} \\
C(x, t)=C_{0}\left\{1-\frac{4}{\pi} \sum_{n=\text { odd }}^{\infty} \frac{1}{n}\left[\exp \left(-n^{2} \pi^{2} D t / 4 l^{2}\right) \sin (n \pi x / l)\right]\right\}
\end{gathered}
$$

where $l$ is the length of the filled region of the tube. The solution of equation (3) converges satisfactorily for small values of $t$ and that of equation (4) for large values, and were employed accordingly, i.e. equation (3) for 1 and $2 \mathrm{~min}$ and equation (4) for longer periods. Derivatives of equation (4) gave the distance (equation 5 ) and time (equation 6) dependent concentration gradients

$$
\begin{gathered}
\frac{\partial C}{\partial x(t)}=-C_{0} \sum_{n=\text { odd }}^{\infty}(2 / l)\left[\exp \left(-n^{2} \pi^{2} D t / 4 l^{2}\right) \cos (n \pi x / 2 l)\right] \\
\frac{\partial C}{\partial t(x)}=-C_{0} \sum_{n=\text { odd }}^{\infty}\left(n \pi d / l^{2}\right)\left[\exp \left(-n^{2} \pi^{2} D t / 4 l^{2}\right) \sin (n \pi x / 2 l)\right]
\end{gathered}
$$

The velocity of band migration, $\mathrm{d} x / \mathrm{d} t$, was given by the ratio of equations (6) to (5). Numerical results were obtained by means of Fortran programs.

\section{RESULTS AND DISCUSSION}

\section{Indicator test on hydrochloric acid diffusion}

Bromophenol blue solution (150 $\mu \mathrm{M}, \mathrm{pH} 6)$ was prepared from freshly distilled water. The carbon dioxide content and consequent buffering were hence negligible. Titration with $\mathrm{HCl}$ was carried out and changes in colour and $\mathrm{pH}$ were noted. A transition from blue to red occurred at 25 to $100 \mu \mathrm{M}-\mathrm{HCl}$. A capillary tube containing the bromophenol blue was placed in a chemotaxis chamber containing $10 \mathrm{mM}-\mathrm{HCl}$ and the position of the blue/red colour boundary was observed. It receded from the mouth of the tube with time (Fig. 1).

The position with time of a $50 \mu \mathrm{M}-\mathrm{HCl}$ concentration in the capillary tube was calculated from equations (3) and (4). The experimentally determined colour transition corresponded well with the calculated diffusion of $\mathrm{HCl}$. The agreement, however, was not perfect - there was an anomalously high reading at $1 \mathrm{~min}$, perhaps due to mixing of solutions when the experiment was set up, and a systematic trend towards low values for the late readings. The latter suggest that equation (4) does not provide a fully accurate description of diffusion - a decrease in diffusion coefficient with fall in concentration may account for the anomaly. The discrepancy will be most marked with high initial concentrations, where the absolute concentration change of repellent is greatest. 


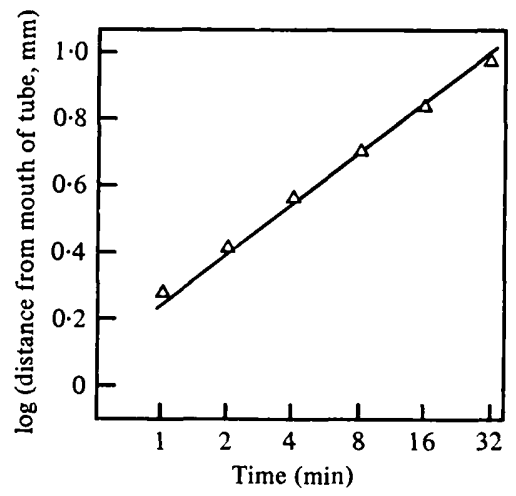

Fig. 1. Recession of the colour boundary with time from the mouth of a capillary tube containing $150 \mu \mathrm{M}$-bromophenol blue placed in a chamber containing $10 \mathrm{mM}-\mathrm{HCl}$. Measured values of the position of the colour boundary are indicated by $\triangle$. The line indicates the calculated diffusion of $\mathrm{HCl}\left(D=0.0033 \mathrm{~mm}^{2} \mathrm{~s}^{-1}\right)$ into the tube as represented for a concentration of $50 \mu \mathrm{M}-\mathrm{HCl}$.

\section{Repulsion of zoospores by cations}

The following cations were tested as chlorides: $\mathrm{H}^{+}, \mathrm{Li}^{+}, \mathrm{Na}^{+}, \mathrm{K}^{+}, \mathrm{Cs}^{+}, \mathrm{Mg}^{2+}, \mathrm{Ca}^{2+}, \mathrm{La}^{3+}$. The solutions were all above $\mathrm{pH} 5$, except for $\mathrm{HCl}$. All cations except $\mathrm{Na}^{+}$caused zoospores to retreat along the capillary tube as a band, initially sharply defined, later more diffuse. At the band edge adjacent to the mouth of the tube zoospores stopped and turned, as if a hard barrier were present, then regained normal motility. The few zoospores that failed to turn soon became non-motile and later lysed. The band edge was very sharply defined in tests with $\mathrm{H}^{+}$, clear with $\mathrm{K}^{+}, \mathrm{Cs}^{+}, \mathrm{Mg}^{2+}$ and $\mathrm{La}^{3+}$ and ill-defined with $\mathrm{Li}^{+}$and $\mathrm{Ca}^{2+}$. The rates at which bands retreated in response to the various cations are indicated in Fig. 2, which shows also the calculated diffusion of the salt or acid into the tube. The highest concentrations of $\mathrm{Na}^{+}$tested caused immobility without bands being observed; presumably the threshold for repulsion, if it occurs, is similar to or greater than that for immobilization (5 mM).

\section{Quantitative relationship between stimulus and response}

The most sharply defined zoospore bands were those obtained with $\mathrm{HCl}$ solutions (Fig. $2 d$ ). The response to $\mathrm{H}^{+}$was hence regarded as the most reliable and the data are here used to evaluate hypotheses concerning the relationship between stimulus and response.

Allen \& Harvey (1974) distinguished two such classes of hypothesis - continuous response and threshold response hypotheses. The former class postulates a continuous response to the repellent over a wide range of intensities, i.e. that the rate of band migration $\mathrm{d} x / \mathrm{d} t$ is some function of repellent concentration $C$ or concentration gradient $\partial C / \partial x$. A continuous response to concentration can, however, be excluded since the $\mathrm{H}^{+}$concentration at the band position calculated from equation (4) remains roughly constant throughout an experiment whereas the rate of band migration changes greatly. A continuous response to the concentration gradient can also be excluded because although the rate of band migration was approximately proportional to the concentration gradient, i.e. $\mathrm{d} x / \mathrm{d} t \propto \partial C / \partial x$, the constant of proportionality depended on the initial concentration (Fig. $3 a$ ). A further possibility is that the rate of band migration is determined by the gradient of logarithmic concentration $(1 / C)(\partial C / \partial x)$, a relationship between stimulus and response found for positive chemotaxis in Escherichia coli (Dahlquist et al., 1972). This possibility must, however, also be dismissed since it does not meet a condition necessary for band formation - that the concentration function at a given time must decrease with distance from the mouth of the tube.

The alternative type of hypothesis is that an all-or-none response occurs when zoospores 


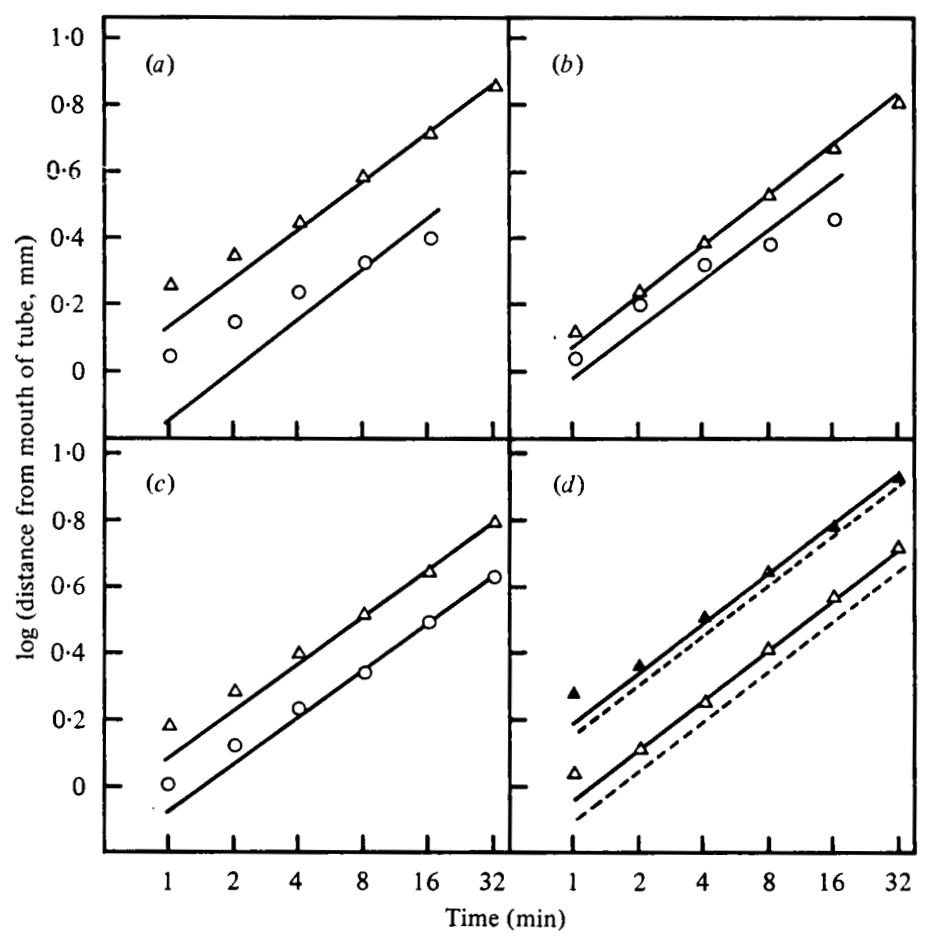

Fig. 2. Retreat of zoospores in capiliary tubes placed in solutions containing different cations. Measured values of the distance of the zoospore band from the mouth of the tube are indicated by $O$ and $\Delta$. The lines indicate the calculated diffusion of the salt into the tube, as represented for particular concentrations; these may be regarded as the threshold concentrations for repulsion (see text). The concentrations of the salts in the chambers, the diffusion coefficients $(D)$ and the threshold concentrations were as follows. (a) $100 \mathrm{~mm}-\mathrm{LiCl}(O), D=0.0012 \mathrm{~mm}^{2} \mathrm{~s}^{-1}$, threshold $5 \mathrm{~mm} ; 100 \mathrm{~mm}-\mathrm{CsCl}(\triangle), D=0.0019 \mathrm{~mm}^{2} \mathrm{~s}^{-1}$, threshold $0.6 \mathrm{~mm}$. (b) $100 \mathrm{mM}^{-\mathrm{CaCl}_{2}}(\bigcirc)$, $D=0.0011 \mathrm{~mm}^{2} \mathrm{~s}^{-1}$, threshold $1.0 \mathrm{mM} ; 100 \mathrm{~mm}-\mathrm{KCl}(\triangle), D=0.0019 \mathrm{~mm}^{2} \mathrm{~s}^{-1}$, threshold $1.2 \mathrm{~mm}$. (c) $17 \mathrm{mM}-\mathrm{MgCl}_{2}(O), D=0.0011 \mathrm{~mm}^{2} \mathrm{~s}^{-1}$, threshold $0.6 \mathrm{~mm} ; 20 \mathrm{~mm}-\mathrm{LaCl}_{\mathbf{8}}(\Delta)$, $D=0.0011 \mathrm{~mm}^{2} \mathrm{~s}^{-1}$, threshold $0.05 \mathrm{~mm}$. (d) $1 \mathrm{~mm}-\mathrm{HCl}(\triangle)$ and $10 \mathrm{~mm}-\mathrm{HCl}(\Delta), D=0.0033$ $\mathrm{mm}^{2} \mathrm{~s}^{-1}$, threshold $150 \mu \mathrm{M}(-)$ or $210 \mu \mathrm{M}(---)$.

encounter a critical threshold concentration or concentration gradient. The latter possibility can be eliminated since the concentration gradient at the band changes with time (Fig. $3 b$ ). The concentration at the band, however, is nearly constant at $150 \mu \mathrm{M}$ for an initial $1 \mathrm{~mm}$ $\mathrm{HCl}$ concentration (Fig. $3 \mathrm{c}$ ), apart from an anomalous low reading at $1 \mathrm{~min}$, an effect that occurred with all cations, and may be associated with setting up the experiment. With an initial $\mathrm{HCl}$ concentration of $10 \mathrm{mM}$ there is a less precise agreement between the position of the zoospore band and the calculated position for $150 \mu \mathrm{M}-\mathrm{HCl}$. There is, however, a close agreement between the band position and the indicator colour change observed in the comparable experiment with $10 \mathrm{~mm}-\mathrm{HCl}$ (Fig. 1). The regression equation (Snedecor \& Cochran, 1974) was $X_{\mathrm{r}(\mathrm{t})}=0.868 X_{\mathrm{i}(\mathrm{t})}+0.11 ; r=0.999$; where $r$ and $i$ refer to repulsion band and indicator change, respectively. The critical correlation coefficient for $P=0.001$ was $r=0.957$. There is hence a very close agreement between a critical $\mathrm{HCl}$ concentration and the position of the zoospore band. We conclude, as did Allen \& Harvey (1974), that the rate of band migration is most readily explained by repulsion of zoospores at a critical $\mathrm{H}^{+}$concentration.

\section{Mechanism of chemoreception}

Zoospores have a net negative charge at the cell surface (Khew \& Zentmyer, 1974). It is hence appropriate to consider the effect of small cations on negatively charged colloidal 


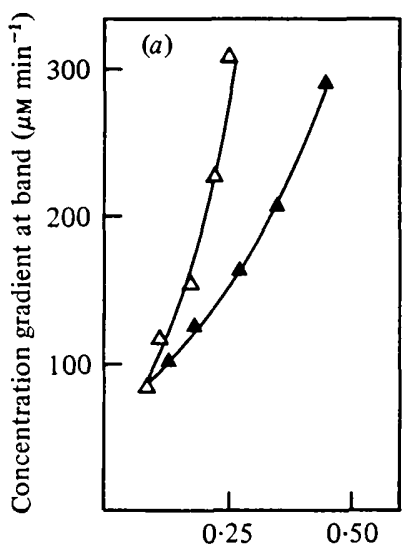

Rate of band migration $(\mathrm{mm} \mathrm{min}-1)$
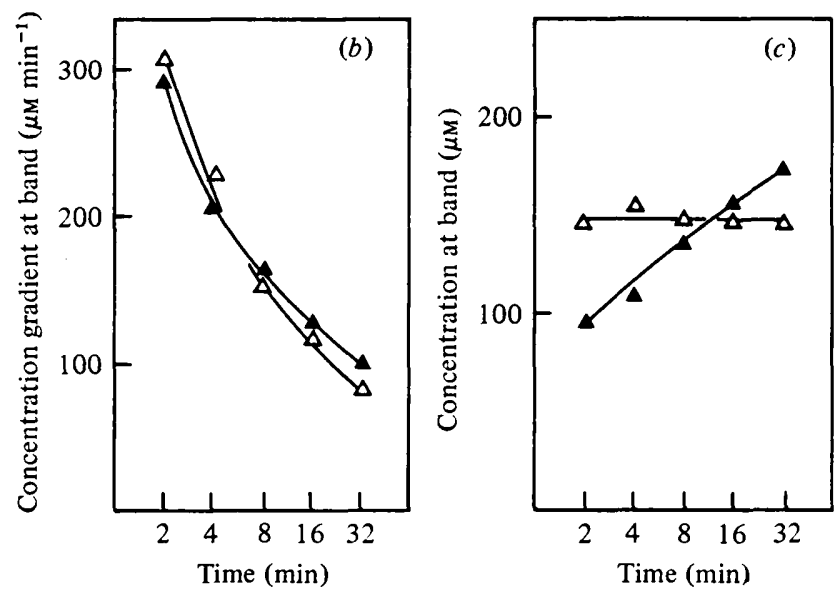

Fig. 3. Evaluation of hypotheses relating stimulus to response for repulsion of zoospores by $\mathbf{H}^{+}$ ions. The concentration of hydrochloric acid in the chamber was $1 \mathrm{~mm}(\triangle)$ or $10 \mathrm{~mm}(\Delta)$.

(a) The possibility that the concentration gradient determines the rate of zoospore band movement is eliminated since the two sets of points do not fall on the same straight line.

(b) The possibility that zoospores respond to a threshold concentration gradient is eliminated since the concentration gradient at the zoospore band position varies with time.

(c) The possibility that zoospores respond to a threshold concentration is accepted as the most likely explanation; the concentration at the band position remains nearly constant at a calculated value of $150 \mu \mathrm{M}$ with an initial concentration of $1 \mathrm{mM}-\mathrm{HCl}$. With an initial concentration of $10 \mathrm{~mm}-\mathrm{HCl}$ the calculated concentration at the zoospore band changes with time. This, however, seems due to the equations providing an inadequate description of diffusion at this initial concentration; dye experiments support the threshold concentration hypothesis (see text).

particles (Adamson, 1976). A negatively charged particle is surrounded by a region known as the electrical double layer (EDL). This consists of a tightly bound layer, about one molecule deep, of positively charged ions adjacent to the particle surface and then a progressively less tightly bound region of positive and negative charges. The greater the total ionic strength and valency of the cations in the surrounding medium, the narrower is the EDL and the closer the cations approach the particle surface. Cations close to the surface can then exchange with cations already bound to the surface, or bind to unoccupied anionic sites. The ease with which this binding occurs depends on the relative ease with which a cation can shed its hydration shell and become more mobile. This is a function of the cation hydration energy, which is indicated by the ionic conductivity of the cation. Approach of cations to the particle surface will cause a reduction of surface potential and in the case of zoospores a change in transmembrane potential (see below). The EDL theory explains the absence of collisions between zoospores, which will be prevented partly by direct repulsion as the two double layers interact, and partly because an osmotic imbalance is created by the higher concentration of ions in the region between the zoospores, causing water to be drawn into this region.

The above treatment implies that the effectiveness of cations as repellents should parallel their ionic conductivities. The agreement is good for monovalent cations but less so for polyvalent cations (Table 1). The latter discrepancy may be due to other properties of the ions; we find (unpublished results) that $\mathrm{Mg}^{2+}$ and $\mathrm{Ca}^{2+}$ are needed for zoospore motility and $\mathrm{La}^{3+}$ is known to block $\mathrm{Ca}^{2+}$ transport in many systems (Young \& Nelson, 1974). The ionic conductivity of a cation is a measure of the mobility of the cation; the mobility is determined by the hydrated ionic radius - the greater the radius, the lower the mobility. Ionic mobility is the basis of the cation exchange potentials exhibited by weakly ionic resins, viz. $\mathrm{H}^{+}>\mathrm{Ca}^{2+}>\mathrm{Mg}^{2+}>\mathrm{Cs}^{+}>\mathrm{K}^{+}>\mathrm{Li}^{+}$(Helfferich, 1962). Ionic mobility is also the 
Table 1. Ionic conductivity and thresholds for zoospore repulsion of various cations

$\begin{array}{lcc}\text { Cation } & \begin{array}{c}\text { Equivalent ionic } \\ \text { conductivity* } \\ (\mathrm{S})\end{array} & \begin{array}{c}\text { Threshold for } \\ \text { repulsion } \\ (\mathrm{mm})\end{array} \\ \mathrm{H}^{+} & 350 & 0 \cdot 15 \\ \mathrm{Cs}^{+} & 77 & 0.6 \\ \mathrm{~K}^{+} & 74 & 2 \cdot 1 \\ \mathrm{Na}^{+} & 50 & >5 \\ \mathrm{Li}^{+} & 39 & 5 \\ \frac{1}{2} \mathrm{Ca}^{2+} & 60 & 1 \cdot 0 \\ \frac{1}{2} \mathrm{Mg}^{2+} & 53 & 0.7 \\ \frac{1}{3} \mathrm{La}^{3+} & 70 & 0.05 \\ & * \text { From Adam (1956). }\end{array}$

basis for the position of cations in the Hofmeister lyotropic series, which indicates the precipitating power of ions on hydrophobic colloids. Allen \& Harvey (1974) noted, with the ions they tested, namely $\mathrm{H}^{+}, \mathrm{K}^{+}, \mathrm{NH}_{4}{ }^{+}$and $\mathrm{Na}^{+}$, that their effectiveness as repellents paralleled their position in the lyotropic series, and concluded that with respect to chemoreception the zoospore acted as an exchange resin with fixed anionic groups of low acid strength. Our results on a wider range of cations are consistent with those of Allen \& Harvey (1974) and our treatment in terms of EDL theory is an extension of theirs. The two studies demonstrate that negative chemotaxis of Phytophthora zoospores to cations is dependent on a general mechanism of cation interaction with a negatively charged membrane. Specific receptors for negative chemotaxis have been reported in Escherichia coli (Tso \& Adler, 1974 ) but the authors did not succeed in finding mutants defective for negative chemotaxis with respect to six of the nine groups of repellents that they examined. These six groups included $\mathrm{H}^{+}, \mathrm{OH}^{-}$, some cations and fatty acids, and it is possible that here too a nonspecific mode of chemoreception occurs.

\section{Mechanism of repulsion}

A change in the charge at the cell surface will, provided the internal charge is unaltered, cause a change in transmembrane potential, either hyperpolarization or depolarization. Since the interior of cells normally has a more negative charge than the cell surface (Harold, 1977), the neutralization of negative charges at the zoospore surface by cations is likely to cause an increase in transmembrane potential, i.e. hyperpolarization. Changes in transmembrane potential are associated with changed flagellum or ciliary activity and turning in many micro-organisms including Escherichia coli (Szmelcman \& Adler, 1976), Rhodospirillum rubrum (Harayama \& Iino, 1977), Rhodopseudomonas sphaeroides (Armitage \& Evans, 1979) and Paramecium tetraurelia (Van Houten, 1979). We therefore suggest that membrane hyperpolarization brings about changed flagellum activity and turning of the Phytophthora zoospore and that one or more acute turns return the zoospore to regions of lower cation concentration which permit depolarization of the cell membrane and smooth swimming.

We wish to thank the Science Research Council for a studentship to one of us (J.N.C.) and Dr J. Adler, Department of Mathematics, Imperial College, and Dr M. E. J. Holwill, Department of Physics, Queen Elizabeth College, for assistance with mathematical problems. 


\section{REFERENCES}

Adam, N. K. (1956). Physical Chemistry. Oxford: Clarendon Press.

Adamson, A. W. (1976). Physical Chemistry of Surfaces, 3rd edn. New York: John Wiley.

Allen, R. N. \& HaRveY, J. D. (1974). Negative chemotaxis of zoospores of Phytophthora cinnamomi. Journal of General Microbiology 84, 28-38.

Armitage, J. P. \& Evans, M. C. W. (1979). Membrane potential changes during chemotaxis of Rhodopseudomonas sphaeroides. FEBS Letters 102 143-146.

Cameron, J. N. \& Carlile, M. J. (1977). Negative geotaxis of zoospores of the fungus Phytophthora. Journal of General Microbiology 98, 599-602.

Cameron, J. N. \& Carlile, M. J. (1978). Fatty acids, aldehydes and alcohols as attractants for zoospores of Phytophthora palmivora. Nature, London 271, 448-449.

Crank, J. (1975). The Mathematics of Diffusion, 2nd edn. Oxford: Clarendon Press.

Dahlquist, F. W., Lovely, P. \& Koshland, D. E. (1972). Quantitative analysis of bacterial migration in chemotaxis. Nature New Biology 236, 120-123.

Harayama, S. \& Iino, T. (1977). Phototaxis and membrane potential in the photosynthetic bacterium Rhodospirillum rubrum. Journal of Bacterio$\log y 131,34-41$.
HAROLD, F. M. (1977). Ion currents and physiological functions in microorganisms. Annual Review of Microbiclogy 31, 181-203.

Helfferich, F. (1962). Ion Exchange. New York: McGraw-Hill.

Khew, K. L. \& Zentmyer, G. A. (1974). Electrotactic responses of seven species of Phytophthora. Phytopathology 64, 500-507.

SNedeCoR, G. W. \& Cochran, W. G. (1974). Statistical Methods, 6th edn. Ames, Iowa: Iowa State University Press.

Szmelcman, S. \& Adler, J. (1976). Change in membrane potential during bacterial chemotaxis. Proceedings of the National Academy of Sciences of the United States of America 73, 4387-4391.

Tso, W. W. \& AdLer, J. (1974). Negative chemotaxis in Escherichia coli. Journal of Bacteriology 118, $560-576$.

Van Houten, J. (1979). Membrane potential changes during chemokinesis in Paramerium. Science 204, 1100-1103.

WEAST, R. C. (1976). Handbook of Chemistry and Physics, 57th edn. Ohio: Chemical Rubber Co.

YounG, L. G. \& Nelson, L. (1974). Calcium ions and control of the motility of sea-urchin spermatozoa. Journal of Reproduction and Fertility 41, $371-378$ 